\title{
Anatomical structures, cell types and biomarkers of the Human Reference Atlas
}

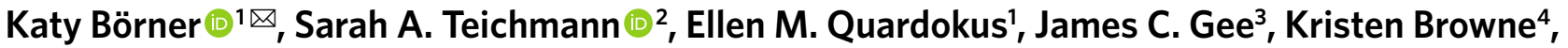 \\ David Osumi-Sutherland ${ }^{5}$, Bruce W. Herr II ${ }^{1}$, Andreas Bueckle ${ }^{(1}{ }^{1}$, Hrishikesh Paul', Muzlifah Haniffa ${ }^{6}$, \\ Laura Jardine ${ }^{6}$, Amy Bernard ${ }^{7}$, Song-Lin Ding ${ }^{8}$, Jeremy A. Miller ${ }^{8}$, Shin Lin ${ }^{9}$, Marc K. Halushka ${ }^{10}$, \\ Avinash Boppana", Teri A. Longacre ${ }^{12}$, John Hickey"12, Yiing Lin ${ }^{13}$, M. Todd Valerius ${ }^{14}$, Yongqun He ${ }^{15}$, \\ Gloria Pryhuber ${ }^{16}$, Xin Sun ${ }^{17}$, Marda Jorgensen ${ }^{18}$, Andrea J. Radtke ${ }^{19}{ }^{19}$, Clive Wasserfalli8, Fiona Ginty ${ }^{20}$, \\ Jonhan $\mathrm{Ho}^{21}$, Joel Sunshine ${ }^{22}$, Rebecca T. Beuschel ${ }^{19}$, Maigan Brusko ${ }^{18}$, Sujin Lee ${ }^{23}$, Rajeev Malhotra ${ }^{14,23}$, \\ Sanjay Jain ${ }^{24,25}$ and Griffin Weber ${ }^{26}$
}

\begin{abstract}
The Human Reference Atlas (HRA) aims to map all of the cells of the human body to advance biomedical research and clinical practice. This Perspective presents collaborative work by members of $\mathbf{1 6}$ international consortia on two essential and interlinked parts of the HRA: (1) three-dimensional representations of anatomy that are linked to (2) tables that name and interlink major anatomical structures, cell types, plus biomarkers (ASCT+B). We discuss four examples that demonstrate the practical utility of the HRA.
\end{abstract}

W ith developments in massively parallel sequencing in bulk and at the single-cell level, researchers can now detect genomic features and genome expression with great precision ${ }^{1}$. Profiling single cells within tissues and organs enables researchers to map the distribution of cells and their developmental trajectories across organs and gives indications as to their functions. In 2021, there are several ongoing, ambitious efforts to map all of the cells in the human body and to create a digital reference atlas of the human body. The final atlas will encompass the three-dimensional (3D) organization of whole organs and thousands of anatomical structures, the interdependencies between trillions of cells, and the biomarkers that characterize and distinguish cell types. It will make the human body computable, supporting spatial and semantic queries run over $3 \mathrm{D}$ structures linked to their scientific terminology and existing ontologies. It will establish a benchmark reference that helps us to understand how the healthy human body works and what changes during ageing or disease.

A network of 16 consortia is contributing to the construction of the HRA based on studies of 30 organs (Fig. 1a) with funding by the National Institutes of Health (NIH, blue) and strong support by the international Human Cell Atlas (HCA, red ${ }^{2,3}$ as well as expert input by reviewers from many different countries.
The 16 consortia include the Allen Brain Atlas ${ }^{4}$, the Brain Research through Advancing Innovative Neurotechnologies Initiative-Cell Census Network Initiative ${ }^{5}$, the Chan Zuckerberg Initiative Seed Networks for HCA ${ }^{2,3,6}$, HCA awards by the EU's Horizon 2020 program, the Genotype-Tissue Expression project ${ }^{7}$, the GenitoUrinary Developmental Molecular Anatomy Project ${ }^{8}$, Helmsley Charitable Trust: Gut Cell Atlas ${ }^{2,3,6,9}$, the Human Tumor Atlas Network ${ }^{10}$, the Human Biomolecular Atlas Program (HuBMAP) ${ }^{11}$, the Kidney Precision Medicine Project (KPMP) $)^{12,13}$, LungMAP ${ }^{14}$, HCA grants from the United Kingdom Research and Innovation Medical Research Council (https://mrc.ukri.org), (Re)building the Kidney ${ }^{15}$, Stimulating Peripheral Activity to Relieve Conditions ${ }^{16}$, The Cancer Genome Atlas ${ }^{17-19}$ and Wellcome funding for HCA pilot projects ${ }^{2,3,6}$. In total, more than 2,000 experts from around the globe are working together to construct an open-source and free-to-use digital HRA using a wide variety of single or multimodal spatially resolved and bulk tissue assays. Imaging methods for anatomical structure segmentation include computed tomography, magnetic resonance imaging or optical coherence tomography (OCT $)^{20}$. Spatially resolved single-cell methods detect metabolites or lipids using high-resolution nanospray desorption electrospray ionization mass spectrometry imaging ${ }^{21}$, proteins using co-detection by

\footnotetext{
'Department of Intelligent Systems Engineering, Indiana University, Bloomington, IN, USA. ${ }^{2}$ Wellcome Sanger Institute, Wellcome Genome Campus, Hinxton, Cambridge, UK. ${ }^{3}$ Department of Radiology, University of Pennsylvania, Philadelphia, PA, USA. ${ }^{4}$ Department of Health and Human Services, National Institute of Allergy and Infectious Diseases, National Institutes of Health, Bethesda, MD, USA. ${ }^{5}$ European Bioinformatics Institute (EMBL-EBI), Wellcome Trust Genome Campus, Cambridge, UK. ${ }^{6}$ Biosciences Institute, Newcastle University, Newcastle upon Tyne, UK. ${ }^{7}$ The Kavli Foundation, Los Angeles, CA, USA. ${ }^{8}$ Allen Institute, Seattle, WA, USA. ${ }^{9}$ Department of Medicine, University of Washington, Seattle, WA, USA. ${ }^{10}$ Department of Pathology, Johns Hopkins University School of Medicine, Baltimore, MD, USA. "Department of Computer Science, Princeton University, Princeton, NJ, USA. ${ }^{12}$ Department of Microbiology and Immunology, Stanford University School of Medicine, Stanford, CA, USA. ${ }^{13}$ Department of Surgery, Washington University in St Louis, St Louis, MO, USA. ${ }^{14}$ Harvard Institute of Medicine, Harvard Medical School, Boston, MA, USA. ${ }^{15}$ Department of Microbiology and Immunology, and Center for Computational Medicine and Bioinformatics, University of Michigan Medical School, Ann Arbor, MI, USA. ${ }^{16}$ Department of Pediatrics, University of Rochester, Rochester, NY, USA. ${ }^{17}$ Biological Sciences, University of California, San Diego, La Jolla, CA, USA. ${ }^{18}$ Department of Pathology, Immunology and Laboratory Medicine, University of Florida, Gainesville, FL, USA. ${ }^{19}$ Center for Advanced Tissue Imaging, Laboratory of Immune System Biology, NIAID, NIH, Bethesda, MD, USA. ${ }^{20}$ Biology and Applied Physics, General Electric Research, Niskayuna, NY, USA. ${ }^{21}$ Department of Dermatology, University of Pittsburgh, Pittsburgh, PA, USA. ${ }^{22}$ Department of Dermatology, Johns Hopkins School of Medicine, Baltimore, MD, USA. ${ }^{23}$ Division of Vascular Surgery and Endovascular Therapy, Massachusetts General Hospital, Boston, MA, USA. ${ }^{24}$ Department of Medicine, Washington University School of Medicine, St Louis, MO, USA. ${ }^{25}$ Department of Pathology and Immunology, Washington University School of Medicine, St Louis, MO, USA. ${ }^{26}$ Department of Biomedical Informatics, Harvard Medical School, Boston, MA, USA. 凶e-mail: katy@indiana.edu
} 


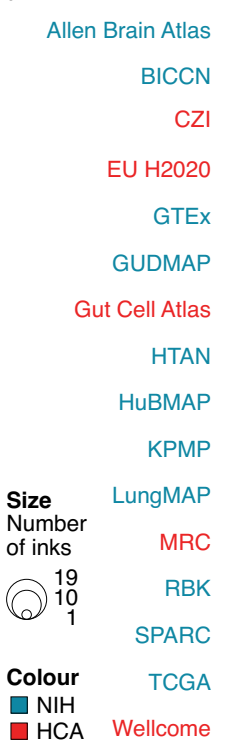
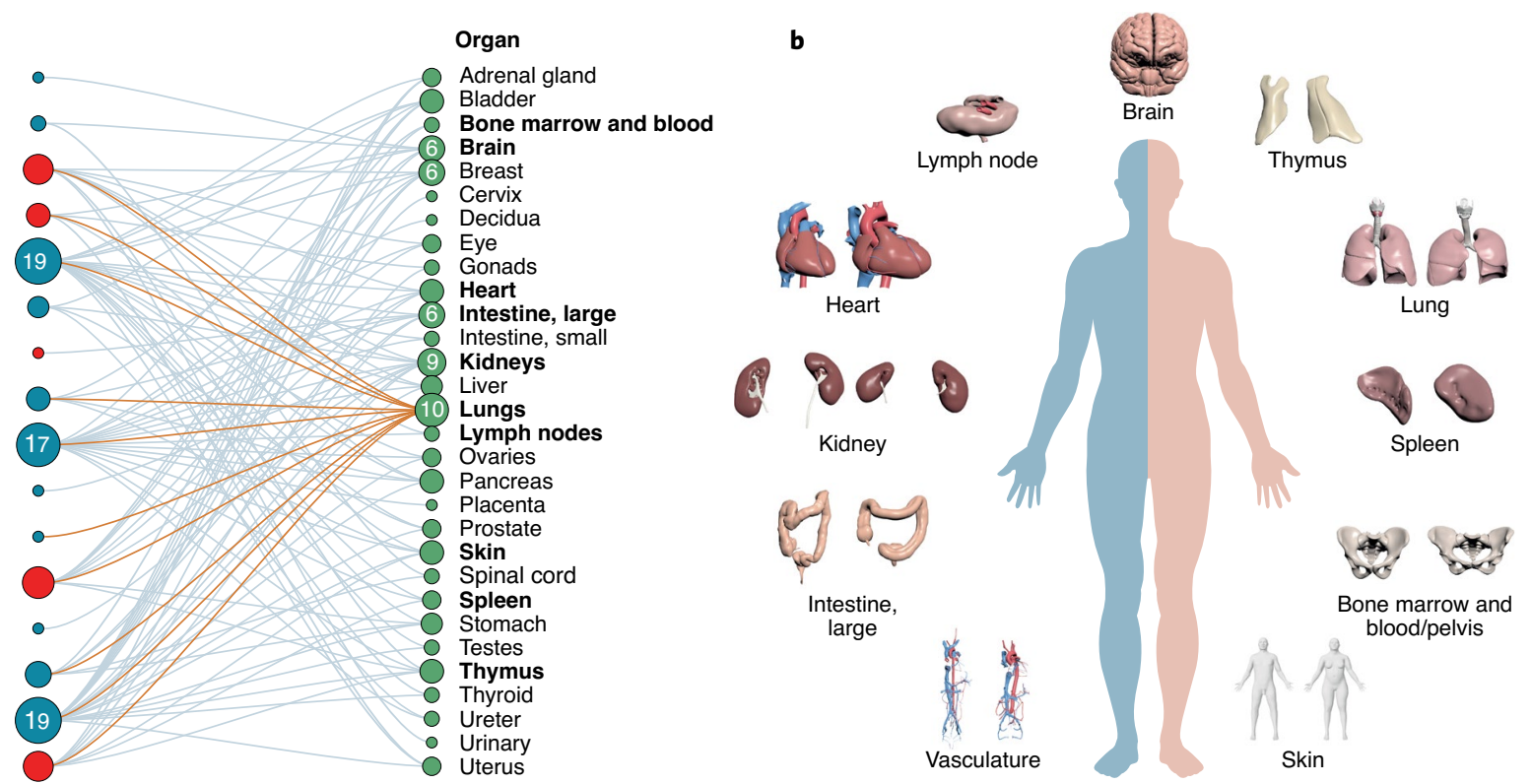

C

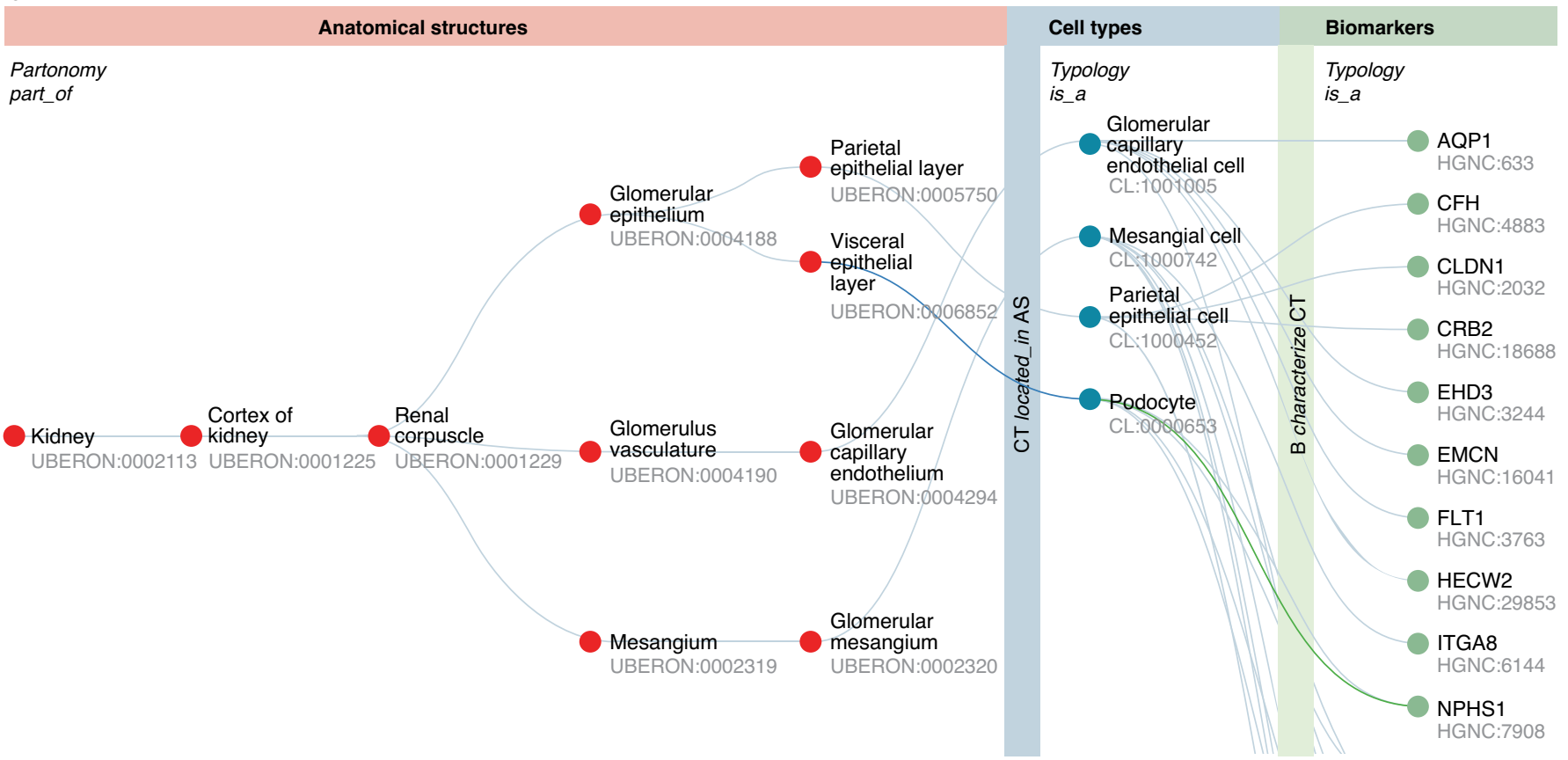

Fig. 1 | Components and construction of the HRA. a, Alphabetical listing of 16 HRA construction efforts (left) linked to the 30 human organs that they study (right). The lungs are studied by ten consortia (orange links). This review focuses on ten organs (bold) plus vasculature. BICCN, Brain Research through Advancing Innovative Neurotechnologies Initiative-Cell Census Network Initiative; CZI, Chan Zuckerberg Initiative; H2020, Horizon 2020; GTEx, Genotype-Tissue Expression project; GUDMAP, GenitoUrinary Developmental Molecular Anatomy Project; HTAN, Human Tumor Atlas Network; MRC, Medical Research Council; RBK, (Re)building the Kidney; SPARC, Stimulating Peripheral Activity to Relieve Conditions; TCGA, The Cancer Genome Atlas. b. The 3D reference objects for major anatomical structures were jointly developed for 11 organs. c, An exemplary ASCT+B table showing anatomical structures (AS) and cell types (CT) and some biomarkers (B) for the glomerulus in the kidneys, annotated with the names of the three entity types (anatomical structures, cell types and biomarkers) and four relationship types (part_of, is_a, located_in and characterize). Note that the is_a relationship exists for cell types and biomarkers.

indexing $^{22}$ or tissue microarray-based immunohistochemistry ${ }^{23}$, simultaneous mRNA and chromatin accessibility using assay for transposase-accessible chromatin with high-throughput sequencing $^{24}$, simultaneous protein and mRNA using cellular indexing of transcriptomes and epitopes by sequencing ${ }^{25}$, and mRNA using multiplexed error robust fluorescent hybridization (MERFISH) ${ }^{26,27}$, Slide-seq ${ }^{28,29}$, Nanostring's GeoMX ${ }^{30}$ or 10x Genomics Visium ${ }^{31}$.
A major challenge in constructing the HRA is combining data generated by the different consortia without a common 'language' shared across them for describing and indexing the data in a spatially explicit and semantically consistent way. Rapid progress in single-cell technologies has led to an explosion of cell-type definitions. When researchers profile the expression of genes, proteins or other biomarkers, they can and do assign different cell types making 


\section{Box 1 | Constructing the ASCT+B tables}

At their core, the ASCT+B tables represent three entity types (anatomical structures, cell types and biomarkers; Fig. 1c) and five relationship types (Fig. 1c and Table 2). Anatomical structures are connected through part_of relationships, creating a partonomy tree. In other words, the connections define whether anatomical structures may be shared parts within an organ or greater anatomical structure. Cell types are linked to other cell types through is_a relationships (for example, $\mathrm{T}$ cell is an immune cell, a cardiac cell is a muscle cell), defining the nature of the cell within a cellular lineage. Biomarkers can be of different types indicated by is_a (for example, they can be of type gene, protein, lipid, or metabolite); they are therefore defined in terms of their molecular nature. A bimodal network links cell types and anatomical structures on the basis of located_in relationships. Note that the same cell type might be located_in multiple anatomical structures, whereas a single anatomical structure might comprise multiple cell types. Biomarkers are linked to the cell types that they characterize through a second bimodal network. Note that one biomarker might be used to characterize multiple cell types, and multiple biomarkers might be required to uniquely characterize one cell type.

The ASCT+B v.1.0 table format makes it possible for human experts to represent the three entity types and five relationship types in a table. The initial set of 11 tables was authored using templated Google sheets. Experts filled in organ-specific tables by entering critical metadata (for example, authors, data and version number) in the top ten rows. Row 11 contains the header of the ASCT+B table, listing the anatomical structures, cell types, biomarkers and publication references from left to right. The table columns can be adjusted as needed (for example, anatomical structures partonomies might have only a few levels ( 5 for kidney), whereas others have many (19 for vasculature)). The v.1.0 format captures two biomarker types: gene markers (BG) and protein markers (BP); proteoforms, lipids and metabolites will be added in v1.1. Publication references document scientific evidence for the existence of the three entity types and their five interrelationships. The remaining rows, starting at row 12 , contain the ASCT $+B$ data-as many rows as there are unique cell types in the organ. Each unique anatomical structure, cell type and biomarker is represented by three columns. The first column lists the domain expert preferred name; whenever possible, this name should match the ontology name in the second column. The third column lists the unique, universally resolvable ontology ID, if available.

To ease table construction and ensure compliance with existing ontologies, a table of organ-specific, non-developmental human data captured in existing formalized ontologies was compiled by ontology experts and provided to the ASCT $+\mathrm{B}$ table authors. The ontologies used initially were Uberon $^{33}$ and Foundational Model of Anatomy for anatomical structures ${ }^{34,35}, \mathrm{CL}^{36}$ for cell types and Human Gene Ontology Nomenclature Committee (HGNC) (https://www.genenames.org/) for biomarkers. Data validation was performed by human experts and computationally by testing expert-curated relationships for validity in Uberon using Ubergraph ${ }^{170}$, a knowledge graph combining mutually referential Open Biological and Biomedical Ontology ontologies, including Cell Ontology and Uberon, and featuring precomputed classifications and relationships. it difficult, if not impossible, to compare results across studies-particularly across organ systems. Indeed, except for the brain ${ }^{32}$, no standards exist for the naming of anatomical structures, cell types and biomarkers. Furthermore, information on what cell types are commonly found in which anatomical structures and what biomarkers best characterize certain cell types is scattered across many ontologies (such as Uberon multi-species anatomy ontology ${ }^{33}$, the Foundational Model of Anatomy Ontology ${ }^{34,35}$, Cell Ontology (CL $)^{36}$ or the Human Gene Ontology Nomenclature Committee (https://www.genenames. org/)) and hundreds of publications about cells identified during development, disease and across multiple species (for example, the atlas efforts for brain ${ }^{37}$, heart ${ }^{38}$, lungs ${ }^{14}$ and kidneys $\left.{ }^{13}\right)$. Some critically important details (such as the morphology and distribution of microanatomical structures or the spatial layout of functionally interdependent cell types) are captured through hand-drawn figures-not digitally-without a shared 3D spatial reference system. The lack of a central, unified benchmark reference framework and language impedes progress in biomedical science as it is challenging or impossible to manage, compare, harmonize or use published data.

\section{Towards a unified reference framework for mapping the human body}

To address these issues, an NIH-HCA-organized meeting in March 2020 brought together leading experts to agree on major ontologies and associated 3D anatomical reference objects and to expand them as needed to capture the healthy human adult body. On the basis of ensuing discussions, more than 50 experts-including physicians, surgeons, anatomists, pathologists, experimentalists and representatives from the various consortia-have agreed on a reference framework to digitally represent relevant knowledge. The framework includes data structures, standard operating procedures and visual user interfaces that can be used by anatomists, pathologists, surgeons and other domain experts to digitize, integrate and analyse massive amounts of heterogeneous data. Experts also agreed on the major ontologies that will be used to create a 'Rosetta Stone' across existing anatomy, cell and biomarker ontologies. As a proof of concept, experts compiled inventories for 11 major organs (Fig. 1a,b): bone marrow and blood plus pelvis reference $\operatorname{organ}^{39-52}$, brain ${ }^{4,32,37,53,54}$, heart ${ }^{55,56}$, large intestine ${ }^{57-68}$, kidneys $^{13,69-77}$, lungs (refs. ${ }^{78-86}$ and Sun, X. \& Morrisey, E., manuscript in preparation), lymph nodes ${ }^{87-97}$, skin ${ }^{98-110}$, spleen ${ }^{89,111-122}$, thymus ${ }^{123-134}$ and vasculature $^{78,135-141}$. For each organ, the experts listed known anatomical structures, the cell types located in these structures and the biomarkers that are commonly used to characterize each cell type (such as gene and protein markers). The results are captured in ASCT+B tables (Fig. 1c) that list and anatomical structures, cell types and biomarker entities, their relationships as well as references to supporting publications. As spatial position and context matter for cell function, the experts collaborated with medical designers to compile 3D, semantically annotated reference objects that cover the anatomically correct size and shape of major anatomical structures in a systematic and computable manner (see the final set for the initial 11 ASCT+B tables in Fig. 1b). Together, the ASCT+B tables and associated $3 \mathrm{D}$ reference objects constitute the HRA.

An initial set of the ASCT $+B$ tables and reference objects have been published (Supplementary Information). They capture data and knowledge that are mandatory for compiling a comprehensive HRA, and they are critically important for facilitating data exchange and collaboration among the 16 consortia and other efforts. They demonstrate how existing knowledge can be captured digitally and reorganized in support of a HRA. The tables and associated 3D reference objects provide an agreed-on framework for experimental data annotation across organs and scales (that is, from whole body to organs, tissues, cell types and biomarkers); they make it possible to compare and integrate data from different assay types (such as single-cell RNA-sequencing (scRNA-seq) ${ }^{142}$ and MERFISH ${ }^{143}$ data) 
Table 1 | The terminology used to describe the HRA framework

\begin{tabular}{|c|c|}
\hline Term & Definition \\
\hline Healthy tissue & $\begin{array}{l}\text { Pathologically unremarkable tissue that can be used to derive the function of healthy cells. What counts as healthy is } \\
\text { influenced by normal ageing, underlying comorbidities and medical interventions before tissue donation. }\end{array}$ \\
\hline Anatomical structures & $\begin{array}{l}\text { Parts of the body in defined locations and regions, including the surface, internal organs and tissues. These structures may be } \\
\text { described by gross or microscopic morphology and include functional tissue units and highly organized cellular ecosystems } \\
\text { (such as alveoli in the lungs). }\end{array}$ \\
\hline Cell types & $\begin{array}{l}\text { Mammalian cells are biological units with a defined function that typically have a nucleus and cytoplasm surrounded by a } \\
\text { membrane. Each cell type may have broad common functions across organs and specialized functions or morphological or } \\
\text { molecular features within each organ or region (for example, epithelial cells in the skin, lungs and kidneys may have shared } \\
\text { and specialized functions according to tissue localization). }\end{array}$ \\
\hline Biomarkers & $\begin{array}{l}\text { Molecular, histological, morphological, radiological, physiological or anatomical features that help to characterize the } \\
\text { biological state of the body. Here we focus on the molecular markers that can be measured to characterize a cell type. }\end{array}$ \\
\hline Ontology & $\begin{array}{l}\text { A set of subject area concepts (here, anatomical structures, cell types and biomarkers), their properties and the relationships } \\
\text { between them. }\end{array}$ \\
\hline Partonomy & A classification hierarchy that represents part-whole relationships. \\
\hline Typology & A classification that represents general types (here, cell types and biomarker types). \\
\hline Bimodal network & A network involving two node types and the relationships between them. \\
\hline Polygon mesh & $\begin{array}{l}\text { A collection of vertices, edges and faces defining the shape of a polyhedral object (here the 3D reference objects that define } \\
\text { anatomical structures). }\end{array}$ \\
\hline $3 \mathrm{D}$ reference object & $\begin{array}{l}\text { Polygon mesh of 3D objects (here, anatomical structures), their object node hierarchy, materials and surface colour/texture, } \\
\text { all in a spatial context. }\end{array}$ \\
\hline 3D collision & The intersection of bounding volumes or polygon meshes detected by an algorithm. \\
\hline Semantic annotation & Ontology term associated with a 3D object that can be used as an object name and to search for or filter the object. \\
\hline Crosswalk & $\begin{array}{l}\text { An ontological mapping of anatomical structure terms in the 3D reference object files to the anatomical structure terms in the } \\
\text { ASCT+B tables. }\end{array}$ \\
\hline
\end{tabular}

for spatially equivalent tissue samples; they are a semantically and spatially explicit reference for healthy tissue and cell identity data that can then be compared against disease settings. Finally, the tables and associated reference organs can be used to evaluate progress on the semantic naming and definition of cell types and their anatomically accurate spatial characterization.

The ultimate goal is an HRA that correctly matches human diversity across populations. However, like the Human Genome Project ${ }^{144,145}$, the initial HRA covers a limited set of donors. For example, the Allen Human Reference Atlas-which is included in the HRA-was derived from one donor. It describes one half of a human brain that is intended to be mirrored to characterize a whole human brain. Most of the other reference organs in the current HRA are constructed from the Visible Human Project (VHP) male and female dataset made available by the National Library of Medicine ${ }^{146}$.

The presented $\mathrm{ASCT}+\mathrm{B}$ tables and $3 \mathrm{D}$ reference objects are designed to be representative of organs and general enough to encompass all donors regardless of variation. All of the listed anatomical structures are expected to be present in a donor, except for sex-specific organs. There exists scientific evidence that the listed cell types are known to be located in the given anatomical structure, and that listed biomarkers are commonly used to identify a cell type. Future versions of the tables are in development and will make it possible to capture and compare data about variations in the size, location, shape and frequency of anatomical structures and cell types across donors. We next describe the data format, design and use of ASCT $+B$ tables and the associated 3D reference objects. The initial HRA presents ten ASCT+B tables interlinked through a vasculature table together with a reference library of major anatomical structures. We discuss four examples that showcase the usage of the initial eleven-organ HRA for tissue registration and exploration, data integration, disease studies and measuring progress towards a more complete HRA. We conclude with a discussion of the next steps and an invitation to collaborate on the construction and usage of a reference atlas for healthy human adults.

\section{ASCT $+\mathrm{B}$ tables}

In 2019, the KPMP project published a first version of the ASCT+B tables to serve as a guide to annotate structures and cell types across multiple technologies in the kidneys ${ }^{13}$. The table data format was expanded and the process for constructing, reviewing and approving the ASCT+B tables was formalized (the key steps are described in Box 1 and the terminology is explained in Tables 1 and 2). Note that the HRA framework is in line with reproducibility best-practices and principles that make data findable, accessible, interoperable and reusable ${ }^{147}$, which are essential for the development of disease atlases, biomedical discovery and, ultimately, health improvements.

In September 2021, there exist 11 ASCT+B tables, version 1.0. These master tables are available for free online ${ }^{148}$. They capture 1,424 anatomical structures, 591 cell types and 1,867 biomarkers. The anatomical structures are linked by 2,543 'part_of' relationships, 4,611 'located_in' relationships between cell types and anatomical structures and 3,708 'characterize' links between biomarkers and cells, supported by 293 unique scholarly publications and 506 web links (Supplementary Information). Like the first maps of our world, the first ASCT $+\mathrm{B}$ tables are imperfect and incomplete (see the 'Limitations' section). However, they digitize and standardize existing data and knowledge by clinicians, pathologists, anatomists and surgeons at the gross anatomical level; biologists, computer scientists and others at the single-cell level; and chemists, engineers and others at the biomarker level.

\section{Three-dimensional reference object library}

The spatial location of cell types within anatomical structures matters, as do the number and types of cells within the same anatomical structure. A 3D reference object library was compiled (key steps are described in Box 2) to capture the size, shape, position and rotation 
Table 2 | ASCT+B relationships and their definitions

\begin{tabular}{ll} 
Relationship & Definition \\
\hline part_of & $\begin{array}{l}\text { The partonomy relationship between anatomical } \\
\text { structures. The anatomical structure partonomy } \\
\text { tree represents part-whole relationships of multiple } \\
\text { smaller anatomical structures inside larger anatomical } \\
\text { structures. }\end{array}$ \\
\hline located_in & $\begin{array}{l}\text { The spatial relationship between a cell type and the } \\
\text { anatomical structures in which these exist. }\end{array}$ \\
characterize & $\begin{array}{l}\text { The relationship between molecular biomarkers and cell } \\
\text { types on the basis of experimental evidence. Typically, } \\
\text { only the top most discriminative biomarkers are listed. }\end{array}$ \\
is_a (CT) & $\begin{array}{l}\text { The typology relationship between cell types (CT). The } \\
\text { classification that groups detailed cell types according } \\
\text { to their common characteristics or lineage into larger } \\
\text { groups (for example, an endothelial cell is a vascular cell } \\
\text { type). }\end{array}$ \\
is_a (B) & $\begin{array}{l}\text { The typology relationship between biomarkers (B). A } \\
\text { classification that groups biomarkers by their chemical } \\
\text { nature (here, gene, protein, proteoform, lipid and } \\
\text { metabolite markers). }\end{array}$ \\
&
\end{tabular}

of major anatomical structures in the organ-specific ASCT+B tables (Box 1, Tables 1 and 2, and Supplementary Information).

In September 2021, there exist 26 organ objects that complement the 11 ASCT+B tables discussed above. Male and female versions exist for all organs, and left and right versions exist for kidneys and lymph nodes. All organs are properly positioned in the male and female bodies and are freely available online ${ }^{149}$. The resulting $3 \mathrm{D}$ reference object library captures a total of 1,185 unique 3D structures (for example, the left female kidney has 11 renal papillae; see the complete listing in the HuBMAP Consortium 3D Reference Object Library ${ }^{149}$ ) with 557 unique Uberon terms, including the name of the organ. Files are provided in the GLB format ${ }^{150}$ and are used in several user interfaces (see the 'Tissue registration and exploration' section).

A 'crosswalk' mapping file links the anatomical structure names listed in the ASCT $+\mathrm{B}$ tables and the terminology used in the 3D reference object library GLB files (see the 'CCF 3D reference object library \& crosswalk' section in the Supplementary Information). This interlinkage of ontology terms (for example, kidney or cortex of kidney; Fig. 1c), and the 3D references objects with well-defined polygon meshes (Table 1) that describe the size and shape of these anatomical structures in $3 \mathrm{D}$ (Fig. 1b) is critical for using 3D collision-detection algorithms for tissue registration and spatial search (see the 'Tissue registration and exploration' section).

\section{How to use the HRA}

The ASCT $+\mathrm{B}$ tables and associated $3 \mathrm{D}$ reference organ objects provide a starting point for the systematic construction of a HRA. They provide common nomenclature for major entities and relationships along with cross-references to existing ontologies and supporting literature. In the following sections, we provide four examples illustrating the value of the tables and associated 3D reference organs: (1) to support experimental tissue data registration and annotation across organs and scales (see the next section); (2) to compare and integrate data from different assay types (see the 'Comparing cell states across different tissues and in disease' section); (3) to compare healthy and disease data (see the 'Understanding disease' section); (4) and to evaluate progress toward the compilation of a comprehensive HRA (see the 'Measuring progress' section).

\section{Box 2 | Designing the 3D reference object library}

To create male and female reference objects for the 11 initial organs, experts collaborated closely with medical designers to develop anatomically correct, vector-based objects that correctly represent human anatomy and are labelled using the ontology terms captured in the ASCT $+\mathrm{B}$ tables.

Data from the VHP male and female dataset, which was made available by the National Library of Medicine ${ }^{146}$, were used to model all of the 3D reference organs except for the brain, large intestine and lymph node. The brain uses the 141 anatomical structures of the 'Allen Human Reference Atlas-3D, 2020' representing one half of the human brain ${ }^{4}$; these structures were mirrored to arrive at a whole human brain (as intended by the brain model authors) and resized to fit the visible human male and female bodies. A 3D model of the male large intestine was provided by A. Kaufman (Stony Brook University) modified to fit into the VHP male body, and used to guide the design of the female large intestine. The lymph node was created using mouse data and the clearing-enhanced 3D method developed by W. Li at the Laboratory of Immune System Biology, National Institute of Allergy and Infectious Diseases, $\mathrm{NIH}^{171}$. Although the size and cellular composition of mouse and human lymph nodes vary, the overall anatomy is well conserved between species.

All models were created using the medical image processing tool 3D Slicer ${ }^{172,173}$ and modelling tools such as ZBrush ${ }^{174}$ and Maya ${ }^{175}$. Files are provided in the Graphics Language Transmission Format (GLB) format ${ }^{150}$, which is a widely used standard file format for 3D scenes and models. GLB formats can be viewed in the free Babylon.js Sandbox (https://sandbox. babylonjs.com/), making it possible for anyone to explore the 3D reference objects using a web browser, without downloading or installing new software.

Tissue registration and exploration. Like any atlas, a HRA is a collection of maps that capture a multiscale $3 \mathrm{D}$ reality. Like other digital maps, it supports panning and zoom, from the whole body (macro scale; metres), to the organ level (meso scale; centimetres), to the level of functional tissue units (such as alveoli in the lungs, crypts in the colon and glomeruli in the kidneys; millimetres), down to the single-cell level (micro scale; micrometres). To be usable, the maps in an HRA must use the same index terms and a unifying topological coordinate system such that cells and anatomical structures in adjacent overlapping maps or at different zoom levels can be uniquely named and properly aligned.

The ASCT $+B$ tables and $3 \mathrm{D}$ reference organs provide a framework for experimental data annotation and exploration across organs and scales-that is, from the entire body down to organs, tissues, cell types and biomarkers. For example, they are used to support the registration of new tissue data as well as spatial and semantic search, browsing and exploration of human tissue data. The HuBMAP CCF Registration User Interface ${ }^{151}$ (RUI; Fig. 2a) and CCF Exploration User Interface (EUI) ${ }^{152}$ (Fig. 2b) are available at the HuBMAP portal ${ }^{153}$. The code for both user interfaces is freely available at GitHub HuBMAP Consortium CCF User Interfaces ${ }^{154}$ (https://github.com/hubmapconsortium/ccf-ui). Several consortia and single investigators have used the stand-alone version of the CCF RUI ${ }^{151}$ to register human tissue samples. Resulting data have been added to the CCF EUI making it possible for data providers and others to explore these tissue datasets in the context of human anatomy, to search and filter for datasets that match certain criteria (such as a specific age, sex and assay type) and to access and download raw data. 
a

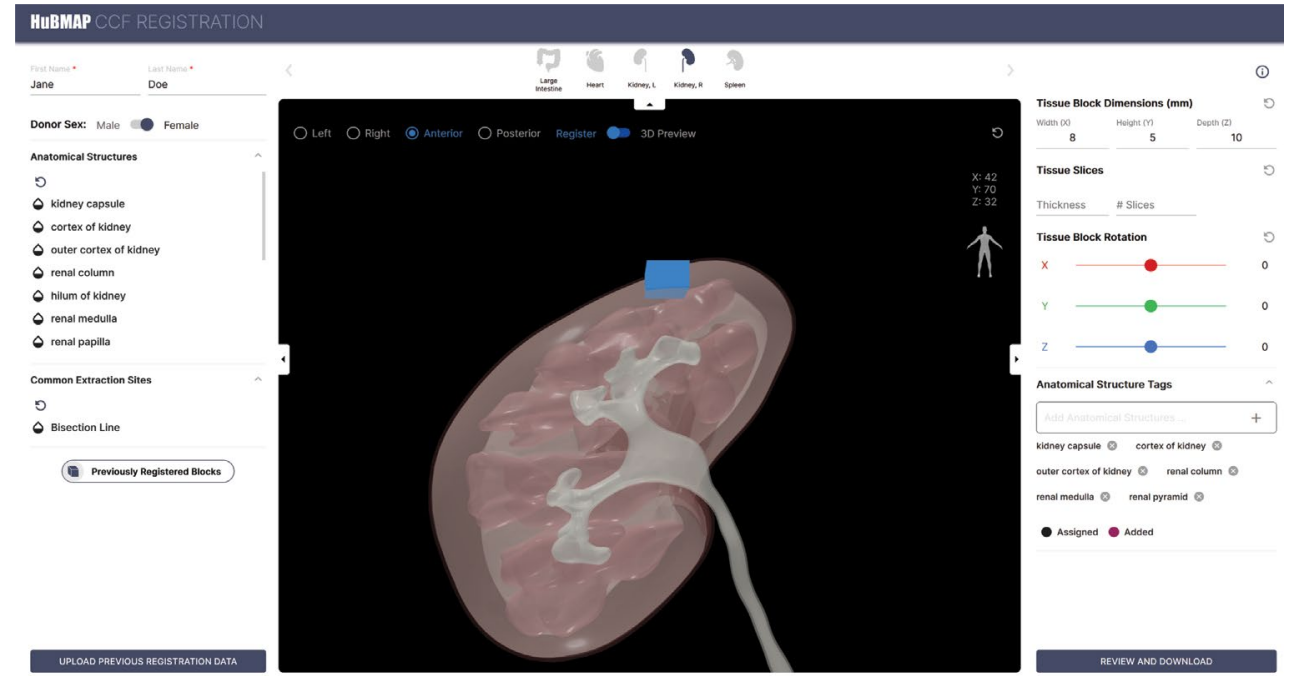

b

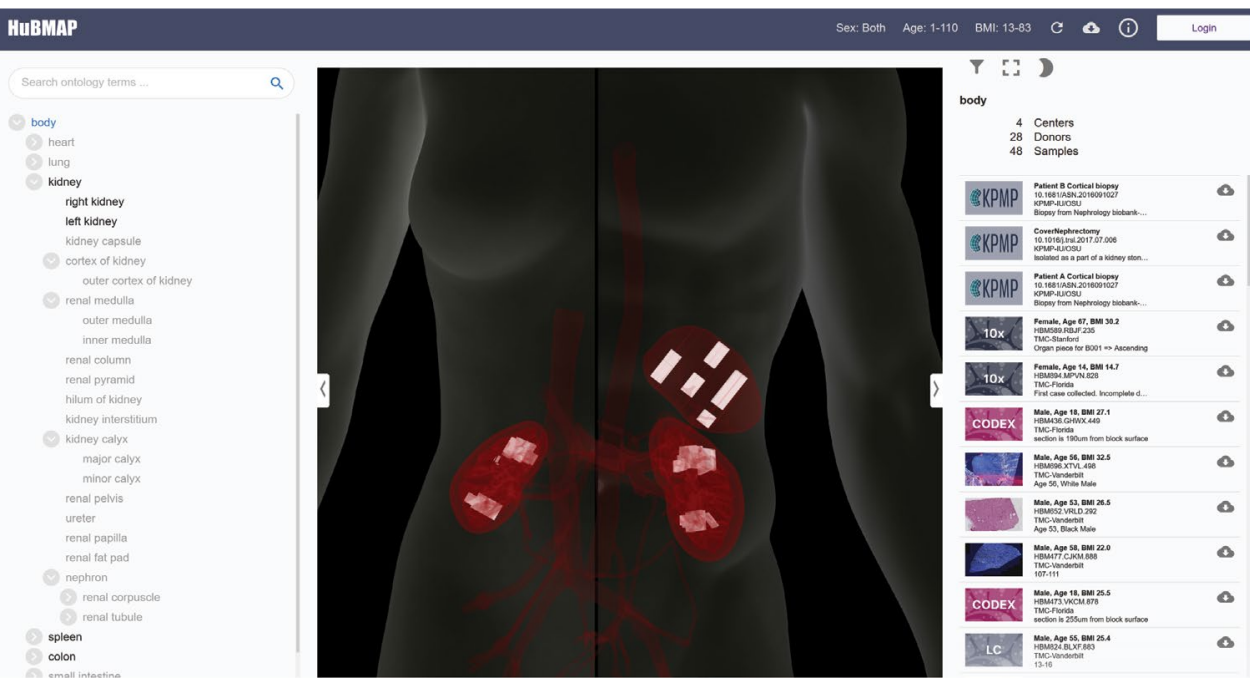

Fig. 2 | Tissue registration and exploration. a, Registration and anatomical structure annotation of tissue data (blue block) in 3D through collision detection in the RUI. A user sizes, positions and rotates tissue blocks, and saves the results in the JavaScript Object Notation (JSON) format. b, Tissue data can be queried, filtered and explored through the EUI. RUI-registered tissue data (white blocks in the spleen and kidneys) can be explored semantically using the anatomical structure partonomy on the left and spatially using the anatomy browser in the middle; a filter at the top right supports subsetting by sex, age, tissue provider and so on. Clicking on a tissue sample on the right links to the Vitessce image viewer ${ }^{169}$.

To make it easy for anyone to explore or contribute HRA data, we developed diverse learning modules as part of the free Visible Human Massive Open Online Course (https:/expand.iu.edu/ browse/sice/cns/courses/hubmap-visible-human-mooc).Thiscourse describes the compilation and coverage of HRA data, demonstrates new single-cell analysis and mapping techniques, and introduces the diverse HRA user interfaces. Delivered entirely online, all coursework can be completed asynchronously to fit busy schedules.

Comparing cell states across different tissues and in disease. The ASCT $+B$ framework provides a look-up table' from the 3D reference models to the unique ontology name and ID for anatomical structures and their cell-type composition across organs that are present in the ASCT+B tables. Researchers can use the tables to determine in what anatomical structure a cell type is commonly located. Specifically, the ASCT+B tables capture information on cells formed within and resident in a specific tissue (such as epithelia and stroma) as well as cells that migrate across tissues (such as immune cells $)^{155}$. For example, immune cells originate primarily in the bone marrow in postnatal life. Adaptive lymphocytes subsequently differentiate and mature in lymphoid tissues such as the thymus and spleen before circulating to non-lymphoid tissues and lymph nodes (Fig. 3a). Thus, these cells recur across the ASCT+B tables in both the lymphoid (bone marrow, thymus, spleen, lymph node) and non-lymphoid (brain, heart, kidney, lung, skin) tissue tables. Existing data support a more nuanced and tissue-specific, ontology-based assignment of blood and immune cells. For example, scRNA-seq enables deep phenotyping of haematopoietic stem cells (HSCs) and haematopoietic stem and progenitor cells (HSPCs) and their differentiated progenies across various tissues. When comparing fetal liver versus thymic cell states (Fig. 3a (right)), a small region of the HSPCs highlighted as lymphoid progenitors is shared across the two organs, indicating cells that have migrated from the liver to the thymus ${ }^{52,125}$. Data integration defines molecules (such as chemokine receptors) that determine tissue residency versus migratory properties. These biomarkers in turn define tissue-resident versus migratory cell states, which can be added to the ASCT $+\mathrm{B}$ tables to refine cellular ontology. 
a

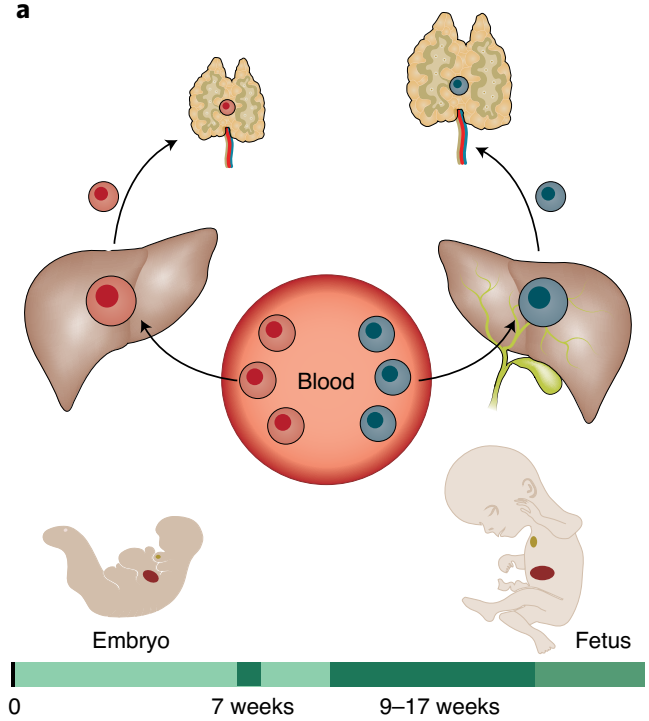

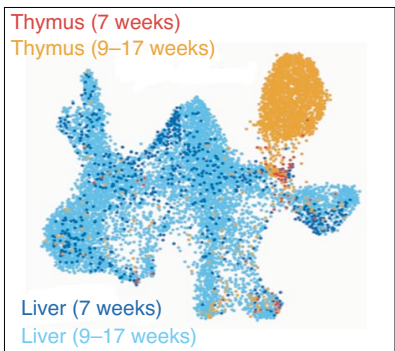
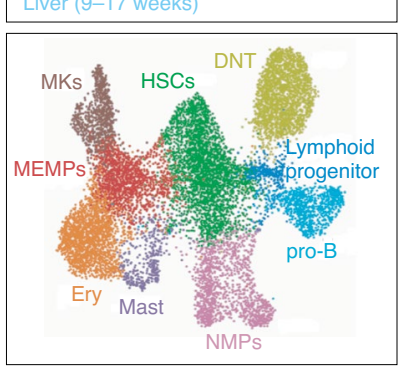
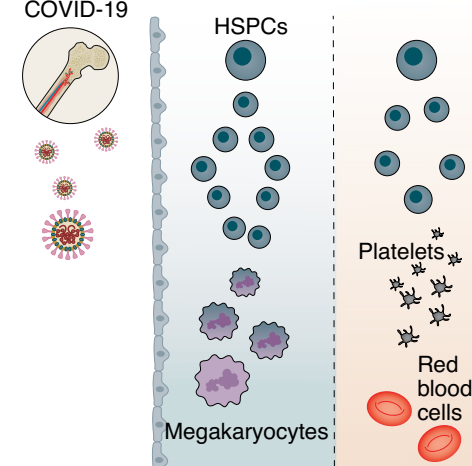

Progenitor cell proportions

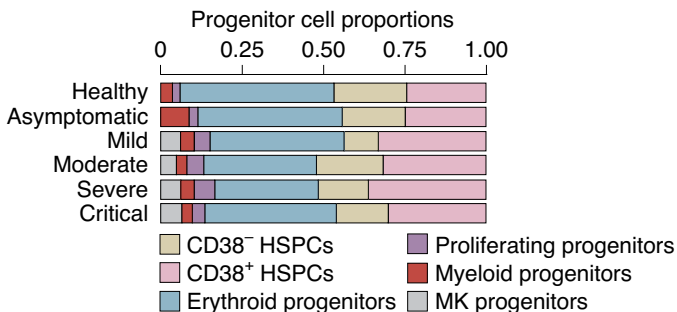

Fig. 3 | Immune cell phenotypes evolve with developmental stage, tissue and disease context. a, HSCs (CL:0000037) migrate from the liver (UBERON:0002107) to the thymus (UBERON:0002370) during embryonic and fetal development. The transcriptomic identity of these HSCs changes throughout pregnancy. The differences in these anatomical structures are shown by the maroon versus blue shading of the HSCs on the left (embryo) and right (fetal) parts of the figure. The scRNA-seq data in the uniform manifold approximation and projection plots are from a published single-cell transcriptomic profile of the thymus across the human lifetime ${ }^{125}$. The top plot shows liver cells (blue) and thymus cells (orange) overlapping, which are labelled lymphoid progenitors in the bottom plot. The other cell populations shown include HSCs; double negative T cells (DNT); megakaryocytes (MKs); megakaryocyte/erythrocyte/mast cell progenitors (MEMPs); pro-B cells; neutrophil-myeloid progenitors (NMPs); mast cells; and erythrocytes (Ery). b. The nature of HSC subsets in the adult blood shifts in health versus COVID-19. HSCs in the blood of patients with COVID-19 (top left) show a megakaryocyte priming bias compared with healthy cells (top right). This is quantified in the histogram from the human thymus single-cell atlas of relative HSPC contributions for different donor/patient cohorts ${ }^{156}$.

A well-annotated healthy HRA can then be used to understand the molecular and cellular alterations in response to perturbations such as infection. For example, data from several single-cell multi-omics studies of patients' blood can be combined to compute the cellular response during COVID-19 pathogenesis, including HSC progenitor states that emerge during disease ${ }^{156}$ (Fig. 3b).

Understanding disease. As a reference for healthy tissue, the ASCT $+B$ tables can be used to identify changes in molecular states in normal ageing or disease. For example, the kidney master table links relevant anatomical structures, cell types and biomarkers to disease and other ontologies for increasing our understanding of disease states. The top significant and specific biomarkers in each reference cell/state cluster might differ during disease or in a cell undergoing repair, regeneration, or in a state of failed or maladaptive repair. Loss of expression or alteration in the cellular distribution of a specific biomarker may provide clues to the underlying disease. The Kidney Precision Medicine Project is working towards ASCT+B tables that characterize disease. Researchers aim to include biomarkers with important physiological roles in maintaining the cellular architecture or function and biomarkers that reveal shifts in cell types that are associated with acute and chronic diseases ${ }^{157}$. Changes in biomarkers in healthy and injured cells provide information about the underlying biological pathways that drive these shifts and therefore provide critical insights into pathogenic mechanisms. For example, the gene NPHS1, which encodes nephrin, is one of the top markers of healthy podocytes and is essential for glomerular function. Mutations in NPHS1 may be found in patients with proteinuria ${ }^{158}$. The kidney ASCT+B table records that the gene biomarker NPHS1 (Fig. 1c (bottom right)) is expressed in the podocytes of the kidney. Ontology suggests injury to podocytes and glomerular function may cause proteinuria (Fig. 4). Ontology IDs provided for anatomical structures, cell types and biomarkers facilitate linkages to clinicopathological knowledge and help to provide broader insights into disease ${ }^{159}$. For example, the ASCT+B kidney master table and single-nucleus RNA-seq atlas data ${ }^{70}$ have been used to characterize diabetic nephropathy disease states by distinguishing the healthy interstitium from a diabetic on ${ }^{160}$. Note that some of the existing data are not at the single-cell level; in these cases, regional data (such as data bounded by tissue blocks registered within reference organs with known anatomical structures, cell types and biomarkers (see the RUI and EUI discussion above)) can be compared to the kidney master table. In summary, ASCT+B tables interlinked with existing ontologies provide a foundation for new data analysis and the functional study of diseases.

Measuring progress. The ASCT+B tables provide objective measures for tracking progress towards an accurate and complete HRA. In particular, if a scholarly publication includes a new ASCT+B table, that table can be compared with existing master tables and the number and type of identical (confirmatory) and different (new) anatomical structures, cell types and biomarkers, as well as their relationships, can be determined. The value of a new data release for reference atlas design can be evaluated in terms of the number and type of new anatomical structures, cell types, biomarkers and their relationships that it contributes. The ASCT+B Reporter ${ }^{161}$ supports the visual exploration and comparison of ASCT+B tables. Table authors and reviewers can use this online tool to upload new 


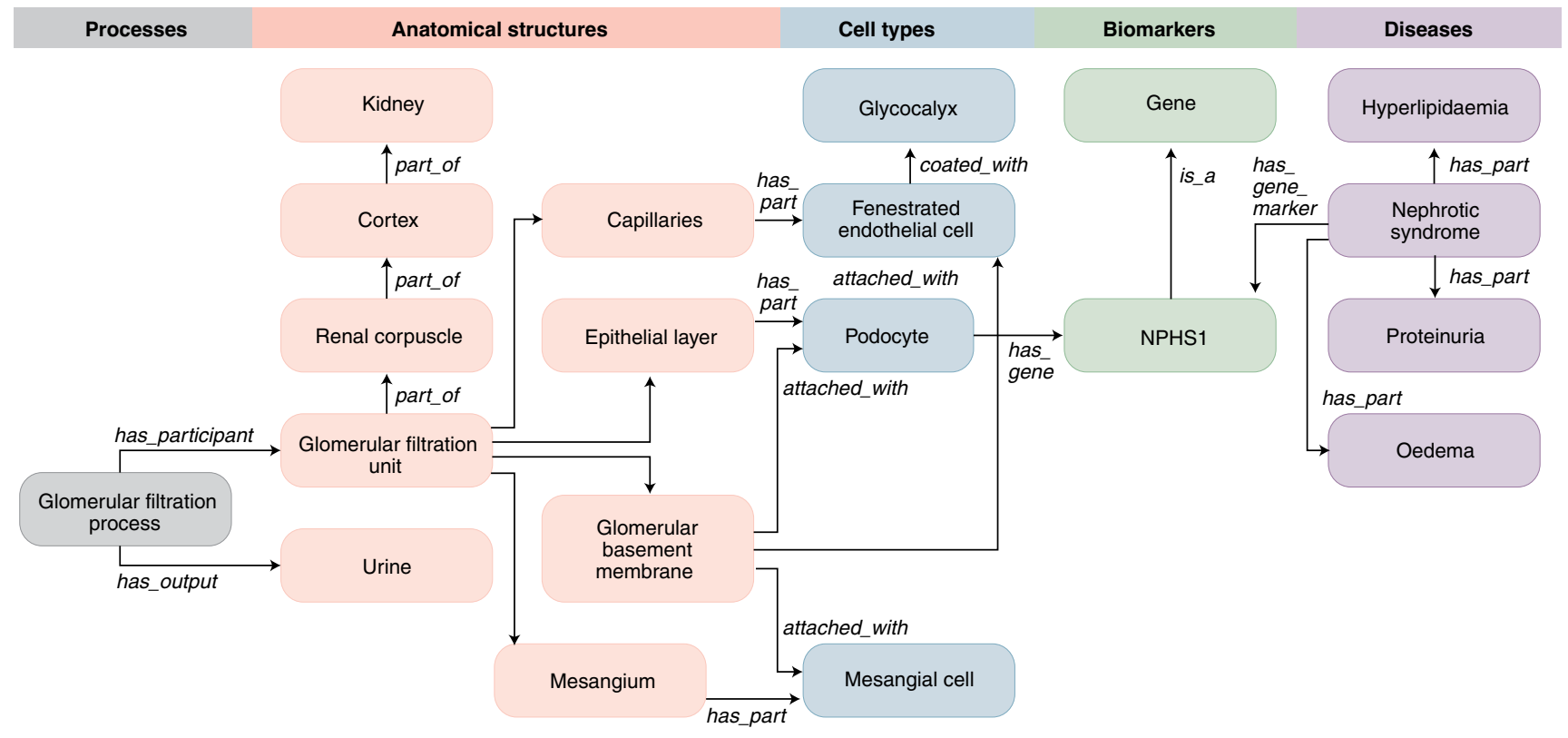

Fig. 4 | Kidney ASCT+B table. Processes, anatomical structures, cell types, biomarkers and disease relevant for understanding proteinuria, in kidney. The example shows how the ASCT+B tables help link clinicopathology information and ontology data.

tables, examine them visually and compare them with existing master tables. Power analysis methods can be run to assess the coverage and completeness of cell states and/or types and decide what tissues and cells should be sampled next ${ }^{139}$ in support of a data-driven experimental design.

As the number of published ASCT $+\mathrm{B}$ tables grows, estimates can be run to determine the likely accuracy of anatomical structures, cell types, biomarkers and relationships. Entities and relationships based on high-quality data or multiple types of scholarly evidence are more likely to be correct compared with those with limited or no evidence. Incomplete data can be easily identified and flagged (for example, anatomical structures with no linkages to cell types and cell types with no biomarkers indicate missing data). Given that the tables disclose who contributes the data and who authors relevant publications, experts on anatomical structures, cell types, biomarkers and their relationships can be identified and invited to further improve the tables.

\section{Limitations}

The current format of the ASCT $+\mathrm{B}$ tables and $3 \mathrm{D}$ reference organs makes them easy to author, review, validate and use across organs and domains of expertise. The ASCT+B Reporter tool ${ }^{161}$ supports the authoring and review of ASCT $+\mathrm{B}$ tables but also the comparison of these data with new datasets. $3 \mathrm{D}$ reference objects can be freely explored in the Babylon.js sandbox in a web browser (https:// sandbox.babylonjs.com/). However, the simplicity of the current tables and organs makes it impossible to fully capture the complexity of the human body. Thus, for each organ-specific table, experts recorded the process that they used to construct the table, which often included simplifying the anatomy to fit within a strict partonomy, making decisions about which cell types and biomarkers had sufficient evidence to be included in the table, or ignoring normal dynamic changes that occur in the organ over time. For several organs, such as the brain, the biomarkers are preliminary and are expected to improve in coverage and robustness in the future.

Biases in sampling with respect to donor demographics (for example, from convenience samples as opposed to using sampling strategies that reflect global demographics), organs (for example, as based on availability of funding) or cell types (for example, due to differential viability or capture efficiency) can be determined and need to be proactively addressed to arrive at an atlas that truly captures healthy human adults. The definition of 'healthy' is expected to evolve; HRA metadata for datasets that were used in the construction of the atlas include information on sex, age and ethnicity, but also comorbidities, making it possible to include or exclude datasets when inclusion criteria change and to recompute the HRA as needed.

Diversity, inclusion, and global scientific equity are major goals for all of the consortia involved in this effort ${ }^{162}$. Authors for the initial set of tables are from the United States and United Kingdom, but many have roots, training and collaborative connections in other parts of the world. Reviewers do not solely come from the United States and United Kingdom. Aiming to overcome the impact of COVID-19 related travel restrictions on collaboration across disciplinary, institutional and cultural boundaries, we organized the HRA panel at the virtual Spatial Biology Europe meeting in April 2021. The panel featured presentations by experts from Asia, Australia and North America. Slides and recordings are available online $^{163}$. In the future, we hope to engage experts from many more countries as well as students from diverse backgrounds.

\section{Outlook}

$\mathrm{ASCT}+\mathrm{B}$ tables in combination with the $3 \mathrm{D}$ reference object library provide a rigorous cross-organ framework for experimental data annotation and exploration from the levels of organs and tissues to the levels of cell types and biomarkers. The construction and validation of the tables are iterative. Initially, ontology and publication data, along with knowledge from organ experts, are codified and unified. Later, experimental datasets are compared with existing master tables to confirm the tables or add to them as needed to capture healthy human tissue data. In the near future, the cell type typology will be expanded from one level to multiple levels, also using data from the evolving set of Azimuth references ${ }^{164,165}$ and in close collaboration with the Cell Ontology curation effort (see ref. ${ }^{166}$ in this issue). This will make it possible to compare anatomical structure partonomy and cell-type typology datasets at different levels of resolution. New organs will be added to the 3D reference library and microanatomical structures, such as glomeruli in the 
kidneys, crypts in the large intestine and alveoli in the lungs will be included.

Efforts are underway to integrate cell-specific protein biomarkers in the ASCT+B tables with well-characterized antibodies for multiplexed antibody-based imaging; the ASCT $+\mathrm{B}$ reporter has already contributed to detect protein biomarkers in situ ${ }^{167,168}$. The goal of these efforts is to generate expertly curated, tissue-specific antibody panels that can be used across consortia in support of a HRA.

The number of anatomical structures, cell types and robust biomarkers will probably increase as new single-cell technologies and computational workflows are developed. Thus, the tables and associated reference objects are a living 'snapshot' of the status of the collective work towards an open, authoritative, computable HRA, against which experimentalists can calibrate their data and to which they can contribute. Future uses of the HRA might include cross-species comparisons or cross-species annotations, cross-tissue/organ comparisons, comparisons of healthy versus common or rare genetic variations, and usage in teaching-expanding widely used anatomy books ${ }^{78,135}$ to the single-cell level.

It will take extensive effort and expertise to arrive at a consensus HRA and to develop methods and user interfaces that use it to advance research and improve human health. Experts interested in contributing to this international and interdisciplinary effort are invited to register at https://iu.col.qualtrics.com/jfe/form/SV bpaBhIr8XfdiNRH to receive regular updates and invites to meetings that aim to advance the construction of the HRA.

Received: 30 January 2021; Accepted: 29 September 2021; Published online: 8 November 2021

\section{References}

1. Chen, G., Ning, B. \& Shi, T. Single-cell RNA-seq technologies and related computational data analysis. Front. Genet. https://doi.org/10.3389/ fgene. 2019.00317 (2019).

2. Rozenblatt-Rosen, O., Stubbington, M. J. T., Regev, A. \& Teichmann, S. A. The human cell atlas: from vision to reality. Nature 574, 187-192 (2017).

3. Regev, A. et al. The Human Cell Atlas. eLife 6, e27041 (2017).

4. Ding, S. L. et al. Comprehensive cellular-resolution atlas of the adult human brain. J. Comp. Neurol. 524, 3127-3481 (2016).

5. Devor, A. et al. The challenge of connecting the dots in the B.R.A.I.N. Neuron 80, 270-274 (2013).

6. Moghe, I., Loupy, A. \& Solez, K. The human cell atlas project by the numbers: relationship to the Banff classification. Am. J. Transpl. 18, 1830 (2018).

7. Lonsdale, J. et al. The Genotype-Tissue Expression (GTEx) project. Nat. Genet. 45, 580-585 (2013)

8. McMahon, A. P. et al. GUDMAP: the genitourinary developmental molecular anatomy project. J. Am. Soc. Nephrol. 19, 667-671 (2008)

9. Elmentaite, R. et al. Cells of the human intestinal tract mapped across space and time. Nature 597, 250-255 (2021).

10. Srivastava, S. et al. The making of a PreCancer Atlas: promises, challenges, and opportunities. Trends Cancer 4, 523-536 (2018).

11. Snyder, M. P. et al. The human body at cellular resolution: the NIH Human Biomolecular Atlas Program. Nature 574, 187-192 (2019).

12. Himmelstein, D. S. et al. Systematic integration of biomedical knowledge prioritizes drugs for repurposing. eLife 6, e26726 (2017).

13. El-Achkar, T. M. et al. A multimodal and integrated approach to interrogate human kidney biopsies with rigor and reproducibility: guidelines from the Kidney Precision Medicine Project. Physiol. Genomics 53, 1-11 (2021).

14. Ardini-Poleske, M. E. et al. LungMAP: the molecular atlas of lung development program. Am. J. Physiol. Lung Cell. Mol. Physiol. 313, L733-L740 (2013).

15. Oxburgh, L. et al. (Re)building a kidney. J. Am. Soc. Nephrol. 28, 1370-1378 (2017).

16. Stimulating Peripheral Activity to Relieve Conditions (SPARC) (NIH, 2020); https://commonfund.nih.gov/sparc

17. Heng, H. H. Q. Cancer genome sequencing the challenges ahead. Bioessays 29, 783-794 (2007)

18. TGCA Research Network Comprehensive genomic characterization defines human glioblastoma genes and core pathways. Nature 455, 1061-1068 (2008).

19. Freire, P. et al. Exploratory analysis of the copy number alterations in glioblastoma multiforme. PLoS ONE 3, e4076 (2008).
20. Aumann, S., Donner, S., Fischer, J. \& Muller, F. in High Resolution Imaging in Microscopy and Ophthalmology: New Frontiers in Biomedical Optics (ed. Bille, J. F.) 59-85. Springer (2019).

21. Yin, R., Burnum-Johnson, K. E., Sun, X., Dey, S. K. \& Laskin, J. High spatial resolution imaging of biological tissues using nanospray desorption electrospray ionization mass spectrometry. Nat. Protoc. 14, 3445-3470 (2019).

22. Goltsev, Y. et al. Deep profiling of mouse splenic architecture with CODEX multiplexed imaging. Cell 174, 968-981 (2018).

23. Uhlén, M. et al. Tissue-based map of the human proteome. Science 347, 1260419 (2015).

24. Buenrostro, J. D. et al. Single-cell chromatin accessibility reveals principles of regulatory variation. Nature 523, 486-490 (2015).

25. Stoeckius, M. et al. Simultaneous epitope and transcriptome measurement in single cells. Nat. Methods 14, 865-868 (2017).

26. Chen, K. H., Boettiger, A. N., Moffitt, J. R., Wang, S. \& Zhuang, X. Spatially resolved, highly multiplexed RNA profiling in single cells. Science 348, aaa6090 (2015).

27. Moffitt, J. R. et al. High-throughput single-cell gene-expression profiling with multiplexed error-robust fluorescence in situ hybridization. Proc. Natl Acad. Sci. USA 113, 11046-11051 (2016).

28. Rodriques, S. G. et al. Slide-seq: a scalable technology for measuring genome-wide expression at high spatial resolution. Science 363 1463-1467 (2019).

29. Asp, M., Bergenstrahle, J. \& Lundeberg, J. Spatially resolved transcriptomes-next generation tools for tissue exploration. Bioessays $\mathbf{4 2}$, e1900221 (2020)

30. Zollinger, D. R., Lingle, S. E., Sorg, K., Beechem, J. M. \& Merritt, C. R. GeoMx RNA Assay: high multiplex, digital, spatial analysis of RNA in FFPE tissue. Methods Mol. Biol. 2148, 331-345 (2020).

31. Visium Spatial Gene Expression (10x Genomics, 2021); https://www. 10xgenomics.com/products/spatial-gene-expression

32. Miller, J. A. et al. Common cell type nomenclature for the mammalian brain. eLife 9, e59928 (2020).

33. Mungall, C. J., Torniai, C., Gkoutos, G. V., Lewis, S. E. \& Haendel, M. A. Uberon, an integrative multi-species anatomy ontology. Genome Biol. 13, R5 (2012).

34. Golbreich, C., Grosjean, J. \& Darmoni, S. J. The foundational model of anatomy in OWL 2 and its use. Artif. Intell. Med. 57, 119-132 (2013).

35. Rosse, C. \& Mejino, J. L. V. A reference ontology for biomedical informatics: the Foundational Model of Anatomy. J. Biomed. Inform. 36, 478-500 (2003).

36. Meehan, T. F. et al. Logical development of the Cell Ontology. BMC Bioinform 12, 6 (2011).

37. Ding, S. L. et al. Allen Human Reference Atlas-3D, 2020 (2021); http:// download.alleninstitute.org/informatics-archive/allen_human_reference_ atlas_3d_2020/version_2021

38. Fonseca, C. G. et al. The Cardiac Atlas Project: an imaging database for computational modeling and statistical atlases of the heart. Bioinformatics 27, 2288-2295 (2011).

39. Géron, A., Werner, J., Wattiez, R., Lebaron, P. \& Mattallana-Surget, S. Deciphering the functioning of microbial communities: shedding light on the critical steps in metaproteomics. Front. Microbiol. 10, 2395 (2019)

40. Manz, M. G., Miyamoto, T., Akashi, K. \& Weissman, I. L. Prospective isolation of human clonogenic common myeloid progenitors. Proc. Natl Acad. Sci. USA 99, 11872-11877 (2002).

41. Fajtova, M., Kovarikova, A., Svec, P., Kankuri, E. \& Sedlak, J. Immunophenotypic profile of nucleated erythroid progenitors during maturation in regenerating bone marrow. Leuk. Lymphoma 54, 2523-2530 (2013).

42. Kawamura, S. et al. Identification of a human clonogenic progenitor with strict monocyte differentiation potential: a counterpart of mouse cMoPs. Immunity 54, 2523-2530 (2017)

43. Mousset, C. M. et al. Comprehensive phenotyping of T cells using flow cytometry. Cytom. A 95, 647-654 (2019).

44. Mello, F. V. et al. Maturation-associated gene expression profiles along normal human bone marrow monopoiesis. Br. J. Haematol. 176, 464-474 (2017).

45. Tomer, A. Human marrow megakaryocyte differentiation: multiparameter correlative analysis identifies von Willebrand factor as a sensitive and distinctive marker for early ( $2 \mathrm{~N}$ and $4 \mathrm{~N})$ megakaryocytes. Blood 104, 2722-2727 (2004).

46. Doulatov, S. et al. Revised map of the human progenitor hierarchy shows the origin of macrophages and dendritic cells in early lymphoid development. Nat. Immunol. 11, 585-593 (2010).

47. Elghetany, M. T., Ge, Y., Patel, J., Martinez, J. \& Uhrova, H. Flow cytometric study of neutrophilic granulopoiesis in normal bone marrow using an expanded panel of antibodies: correlation with morphologic assessments. J. Clin. Lab. Anal. 18, 36-41 (2004). 
48. Szabo, P. A. et al. Single-cell transcriptomics of human T cells reveals tissue and activation signatures in health and disease. Nat. Commun. 10, 4706 (2019).

49. Kaminski, D. A., Wei, C., Qian, Y., Rosenberg, A. F. \& Sanz, I. Advances in human B cell phenotypic profiling. Front. Immunol. 3, 302 (2012).

50. Hay, S. B., Ferchen, K., Chetal, K., Grimes, H. L. \& Salomonis, N. The Human Cell Atlas bone marrow single-cell interactive web portal. Exp. Hematol. 68, P51-P61 (2018).

51. Clavarino, G. et al. Novel strategy for phenotypic characterization of human B lymphocytes from precursors to effector cells by flow cytometry PLoS ONE 11, e0162209 (2016).

52. Popescu, D. M. et al. Decoding human fetal liver haematopoiesis. Nature 574, 365-371 (2019)

53. Hodge, R. D. et al. Conserved cell types with divergent features in human versus mouse cortex. Nature 573, 61-68 (2019).

54. Hawrylycz, M. J. et al. An anatomically comprehensive atlas of the adult human brain transcriptome. Nature 489, 391-399 (2012).

55. Litviňuková, M. et al. Cells of the adult human heart. Nature 588, 466-472 (2020).

56. Tucker, N. R. et al. Transcriptional and cellular diversity of the human heart. Circulation 142, 466-482 (2020).

57. Giannasca, P. J., Giannasca, K. T., Leichtner, A. M. \& Neutra, M. R. Human intestinal M cells display the sialyl Lewis A antigen. Infect. Immun. 67, 946-953 (1999).

58. Buettner, M. \& Lochner, M. Development and function of secondary and tertiary lymphoid organs in the small intestine and the colon. Front. Immunol. 7, 342 (2016).

59. Hoyle, C. H. \& Burnstock, G. Neuronal populations in the submucous plexus of the human colon. J. Anat. 166, 7-22 (1989).

60. Westerhoff, M. \& Greeson, J. in Histology for Pathologists (ed. Mills, S.) Ch. 24, 640-663 (Wolters Kluwer, 2019).

61. Azzali, G. Structure, lymphatic vascularization and lymphocyte migration in mucosa-associated lymphoid tissue. Immunol. Rev. 195, 178-189 (2003).

62. Furness, J. B., Callaghan, B. P., Rivera, L. R. \& Cho, H. J. The enteric nervous system and gastrointestinal innervation: integrated local and central control. Adv. Exp. Med. Biol. 817, 39-71 (2014).

63. Arai, T. \& Kino, I. Morphometrical and cell kinetic studies of normal human colorectal mucosa. Comparison between the proximal and the distal large intestine. Acta Pathol. Jpn 39, 725-730 (1989).

64. Fenton, T. M. et al. Immune profiling of human gut-associated lymphoid tissue identifies a role for isolated lymphoid follicles in priming of region-specific immunity. Immunity 52, 557-570 (2020).

65. Habowski, A. N. et al. Transcriptomic and proteomic signatures of stemness and differentiation in the colon crypt. Commun. Biol. 3, 453 (2020).

66. Lundqvist, C., Baranov, V., Hammarström, S., Athlin, L. \& Hammarström, M. L. Intra-epithelial lymphocytes. Evidence for regional specialization and extrathymic T cell maturation in the human gut epithelium. Int. Immunol. 7, 1473-1487 (1995)

67. Lockyer, M. G. \& Petras, R. E. in Histology for Pathologists (ed. Mills, S.) Ch. 25, 664-676 (Wolters Kluwer, 2019).

68. Pittman, M. E. \& Yantiss, R. K. in Histology for Pathologists (ed. Mills, S.) Ch. 26, 677-691 (Wolters Kluwer, 2019).

69. Kriz, W. \& Bankir, L. A standard nomenclature for structures of the kidney. The Renal Commission of the International Union of Physiological Sciences (IUPS). Kidney Int. 33, 1-7 (1988).

70. Lake, B. B. et al. A single-nucleus RNA-sequencing pipeline to decipher the molecular anatomy and pathophysiology of human kidneys. Nat. Commun. 10, 2832 (2019).

71. Barry, D. M. et al. Molecular determinants of nephron vascular specialization in the kidney. Nat. Commun. 10, 5705 (2019).

72. Menon, R. et al. Single cell transcriptomics identifies focal segmental glomerulosclerosis remission endothelial biomarker. JCI Insight 5, e133267 (2020).

73. Ransick, A. et al. Single-cell profiling reveals sex, lineage, and regional diversity in the mouse kidney. Dev. Cell 51, 399-413 (2019).

74. Limbutara, K., Chou, C. L. \& Knepper, M. A. Quantitative proteomics of all 14 renal tubule segments in rat. J. Am. Soc. Nephrol. 31, 1255-1266 (2020).

75. Kuppe, C. et al. Decoding myofibroblast origins in human kidney fibrosis Nature 589, 281-286 (2021).

76. Stewart, B. J. et al. Spatiotemporal immune zonation of the human kidney. Science 365, 1461-1466 (2019).

77. Kirita, Y., Wu, H., Uchimura, K., Wilson, P. C. \& Humphreys, B. D. Cell profiling of mouse acute kidney injury reveals conserved cellular responses to injury. Proc. Natl Acad. Sci. USA 117, 15874-15883 (2020).

78. Standring, S. Gray's Anatomy: The Anatomical Basis of Clinical Practice (Elsevier, 2016)

79. Haefeli-Bleuer, B. \& Weibel, E. R. Morphometry of the human pulmonary acinus. Anat. Rec. 220, 401-414 (1988)
80. Whitsett, J. A., Kalin, T. V., Xu, Y. \& Kalinichenko, V. V. Building and regenerating the lung cell by cell. Physiol. Rev. 99, 513-554 (2019).

81. Plasschaert, L. W. et al. A single-cell atlas of the airway epithelium reveals the CFTR-rich pulmonary ionocyte. Nature 560, 377-381 (2018).

82. $\mathrm{Xu}$, Y. et al. Single-cell RNA sequencing identifies diverse roles of epithelial cells in idiopathic pulmonary fibrosis. JCI Insight 1, e90558 (2016).

83. Adams, T. S. et al. Single-cell RNA-seq reveals ectopic and aberrant lung-resident cell populations in idiopathic pulmonary fibrosis. Sci. $A d v$. 6, eaba1983 (2020)

84. Wang, A. et al. Single-cell multiomic profiling of human lungs reveals cell-type-specific and age-dynamic control of SARS-CoV2 host genes. eLife 9, e62522 (2020).

85. Travaglini, K. J. et al. A molecular cell atlas of the human lung from single-cell RNA sequencing. Nature 587, 619-625 (2020).

86. Deprez, M. et al. A single-cell atlas of the human healthy airways. Am. J. Respir. Crit. Care Med. 202, 1636-1645 (2019).

87. Medeiros, L. J. et al. in Tumors of the Lymph Node and Spleen Ch. 1 (American Registry of Pathology, 2017).

88. Medeiros, L. J. et al. Tumors of the Lymph Node and Spleen (American Registry of Pathology, 2017).

89. O’Malley, D. P., George, T. I., Orazi, A. \& Abbondanzo, S. L. in Benign and Reactive Conditions of Lymph Node and Spleen Ch. 1 (American Registry of Pathology, 2009).

90. Angel, C. E. et al. Distinctive localization of antigen-presenting cells in human lymph nodes. Blood 113, 1257-1267 (2009).

91. James, K. R. et al. Distinct microbial and immune niches of the human colon. Nat. Immunol. 21, 343-353 (2020).

92. Link, A. et al. Association of T-zone reticular networks and conduits with ectopic lymphoid tissues in mice and humans. Am. J. Pathol. 178, 1662-1675 (2011).

93. Park, S. M. et al. Mapping the distinctive populations of lymphatic endothelial cells in different zones of human lymph nodes. PLOS ONE 9, e106814 (2014).

94. Xiang, M. et al. A single-cell transcriptional roadmap of the mouse and human lymph node lymphatic vasculature. Front. Cardiovasc. Med. 7, 52 (2020).

95. Takeda, A. et al. Single-cell survey of human lymphatics unveils marked endothelial cell heterogeneity and mechanisms of homing for neutrophils. Immunity 51, 561-572 (2019).

96. Kunicki, M. A., Hernandez, L. C. A., Davis, K. L., Bacchetta, R. \& Roncarolo, M. G. Identity and diversity of human peripheral Th and T regulatory cells defined by single-cell mass cytometry. J. Immunol. 200 , 336-346 (2018).

97. Pusztaszeri, M. P., Seelentag, W. \& Bosman, F. T. Immunohistochemical expression of endothelial markers CD31, CD34, von Willebrand factor, and Fli-1 in normal human tissues. J. Histochem. Cytochem. 54, 385-395 (2006).

98. Reynolds, G. et al. Developmental cell programs are co-opted in inflammatory skin disease. Science 371, eaba6500 (2021).

99. Dyring-Anderson, B. et al. Spatially and cell-type resolved quantitative proteomic atlas of healthy human skin. Nat. Commun. 11, 5587 (2020).

100. Fuchs, E. Keratins and the skin. Annu. Rev. Cell Dev. Biol. 11, 123-153 (1995).

101. Nestle, F. O., Meglio, P. D., Qin, J. Z. \& Nickoloff, B. J. Skin immune sentinels in health and disease. Nat. Rev. Immunol. 9, 679-691 (2009).

102. Laverdet, B. et al. Skin innervation: important roles during normal and pathological cutaneous repair. Histol. Histopathol. 30, 875-892 (2015).

103. Ryan, T. J. The blood vessels of the skin. J. Invest. Dermatol. 67, 110-118 (1976).

104. Popescu, D. M. A Single Cell Atlas of Adult Healthy, Psoriatic and Atopic Dermatitis Skin (2021); https://developmentcellatlas.ncl.ac.uk/datasets/ hca_skin_portal

105. Bos, J. D. et al. The skin immune system (SIS): distribution and immunophenotype of lymphocyte subpopulations in normal human skin. J. Invest. Dermatol. 88, 569-573 (1987)

106. Schweizer, J. et al. New consensus nomenclature for mammalian keratins. J. Cell Biol. 174, 169-174 (2006).

107. Eberl, G., Colonna, M., Di Santo, J. P. \& McKenzie, A. N. J. Innate lymphoid cells: a new paradigm in immunology. Science 348, aaa6566 (2015).

108. Ali, N. \& Rosenblum, M. D. Regulatory T cells in skin. Immunology 152 , 372-381 (2017).

109. Huber, W. E. et al. A tissue-restricted cAMP transcriptional response: SOX10 modulates alpha-melanocyte-stimulating hormone-triggered expression of microphthalmia-associated transcription factor in melanocytes. J. Biol. Chem. 278, 45224-45230 (2003).

110. Haniffa, M., Gunawan, M. \& Jardine, L. Human skin dendritic cells in health and disease. J. Dermatol. Sci. 77, 85-92 (2015).

111. Cesta, M. F. Normal structure, function, and histology of the spleen. Toxicol. Pathol. 34, 455-465 (2006). 
112. Madissoon, E. et al. scRNA-seq assessment of the human lung, spleen, and esophagus tissue stability after cold preservation. Genome Biol. 21, 1 (2019)

113. Pack, M. et al. DEC-205/CD205 ${ }^{+}$dendritic cells are abundant in the white pulp of the human spleen, including the border region between the red and white pulp. Immunology 123, 438-446 (2008).

114. Steiniger, B. S. Human spleen microanatomy: why mice do not suffice. Immunology 145, 334-346 (2015)

115. Steiniger, B. S., Seiler, A., Lampp, K., Wilhelmi, V. \& Stachniss, V. B lymphocyte compartments in the human splenic red pulp: capillary sheaths and periarteriolar regions. Histochem. Cell Biol. 141, 507-518 (2014).

116. Steiniger, B. S., Stachniss, V., Schwarzbach, H. \& Barth, P. J. Phenotypic differences between red pulp capillary and sinusoidal endothelia help localizing the open splenic circulation in humans. Histochem. Cell Biol. 128, 391-398 (2007).

117. Qiu, J. et al. The characteristics of vessel lining cells in normal spleens and their role in the pathobiology of myelofibrosis. Blood Adv. 2, 1130-1145 (2018).

118. Lewis, S. M., Williams, A. \& Eisenbarth, S. C. Structure and function of the immune system in the spleen. Sci. Immunol. 4, eaau6085 (2019).

119. Mittag, D. et al. Human dendritic cell subsets from spleen and blood are similar in phenotype and function but modified by donor health status. J. Immunol. 186, 6207-6217 (2011).

120. Cheng, H. W. et al. Origin and differentiation trajectories of fibroblastic reticular cells in the splenic white pulp. Nat. Commun. 10, 1739 (2019).

121. Van Krieken, J. H. J. M. \& Te Velde, J. Immunohistology of the human spleen: an inventory of the localization of lymphocyte subpopulations. Histopathology 10, 285-294 (1986).

122. Van Krieken, J. H. J. M., Te Velde, J., Leenheers-Binnendijk, L. \& Van de Velde, C. J. H. The human spleen; a histological study in splenectomy specimens embedded in methylmethacrylate. Histopathology $\mathbf{9}$, 571-585 (1985).

123. Bautista, J. L. et al. Single-cell transcriptional profiling of human thymic stroma uncovers novel cellular heterogeneity in the thymic medulla. Nat. Commun. 12, 1096 (2021).

124. Haynes, B. F. The human thymic microenvironment. Adv. Immunol. 36 87-142 (1984).

125. Park, J. E. et al. A cell atlas of human thymic development defines T cell repertoire formation. Science 367, eaay3224 (2020).

126. Pearse, G. Normal structure, function and histology of the thymus. Toxicol. Pathol. 34, 504-514 (2006).

127. Suster, S. \& Rosai, J. Histology of the normal thymus. Am. J. Surg. Pathol. 14, 284-303 (1990)

128. Mignini, F. et al. Neuro-immune modulation of the thymus microenvironment (Review). Int. J. Mol. Med. 33, 1392-1400 (2014)

129. Stoeckle, C. et al. Isolation of myeloid dendritic cells and epithelial cells from human thymus. J. Vis. Exp. 79, e50951 (2013).

130. Marcovecchio, G. E. et al. Thymic epithelium abnormalities in DiGeorge and Down syndrome patients contribute to dysregulation in T cell development. Front. Immunol. 10, 447 (2019).

131. Wakimoto, T. et al. Identification and characterization of human thymic cortical dendritic macrophages that may act as professional scavengers of apoptotic thymocytes. Immunobiology 213, 837-847 (2008).

132. Lavaert, $M$. et al. Integrated scRNA-seq identifies human postnatal thymus seeding progenitors and regulatory dynamics of differentiating immature thymocytes. Immunity 52, 1088-1104 (2020).

133. Nuñez, S. et al. The human thymus perivascular space is a functional niche for viral-specific plasma cells. Sci. Immunol. 1, eaah4447 (2016).

134. Bendriss-Vermare, N. et al. Human thymus contains IFN- $\alpha$-producing CD11c $\mathrm{c}^{-}$, myeloid $\mathrm{CD} 11 \mathrm{c}^{+}$, and mature interdigitating dendritic cells. J. Clin. Invest. 107, 835-844 (2001).

135. Netter, F. H. Atlas of Human Anatomy 7th edn (Elsevier, 2019).

136. Kandathil, A. \& Chamarthy, M. Pulmonary vascular anatomy \& anatomical variants. Cardiovasc. Diagn. Ther. 8, 201-207 (2018).

137. Perlmutter, D. \& Rhoton, A. L. Jr Microsurgical anatomy of the distal anterior cerebral artery. J. Neurosurg. 49, 204-228 (1978).

138. Hacein-Bey, L. et al. The ascending pharyngeal artery: branches, anastomoses, and clinical significance. AJNR Am. J. Neuroradiol. 23 , 1246-1256 (2002).

139. Vuong, S. M., Jeong, W. J., Morales, H. \& Abruzzo, T. A. Vascular diseases of the spinal cord: infarction, hemorrhage, and venous congestive myelopathy. Semin. Ultrasound CT MR 37, 466-481 (2016)

140. Picel, A. C., Hsieh, T. C., Shapiro, R. M., Vezeridis, A. M. \& Isaacson, A. J Prostatic artery embolization for benign prostatic hyperplasia: patient evaluation, anatomy, and technique for successful treatment. Radiographics 39, 1526-1548 (2019).

141. Vummidi, D. et al. Pseudolesion in segment IV A of the liver from vein of Sappey secondary to SVC obstruction. Radiol. Case Rep. 5, 394 (2015)

142. Tang, F. et al. mRNA-Seq whole-transcriptome analysis of a single cell. Nat. Methods 6, 377-382 (2009).
143. Moffitt, J. R. et al. Molecular, spatial, and functional single-cell profiling of the hypothalamic preoptic region. Science https://doi.org/10.1126/science. aau5324 (2018).

144. International Human Genome Sequencing (IHGS) Consortium Initial sequencing and analysis of the human genome. Nature 409, 860-921 (2001)

145. Venter, J. C. et al. The sequence of the human genome. Science 291, 1304-1351 (2001)

146. Visible Human Project (VHP) Data Sets (NLM, 2020); https://www.nlm.nih. gov/databases/download/vhp.html

147. Fair Principles (Go Fair, 2021); https://www.go-fair.org/fair-principles

148. CCF Anatomical Structures, Cell Types and Biomarkers (ASCT+B) Tables (HuBMAP Consortium, 2021); https://hubmapconsortium.github.io/ccf/ pages/ccf-anatomical-structures.html

149. CCF 3D Reference Object Library (HuBMAP Consortium, 2021); https://hubmapconsortium.github.io/ccf/pages/ccf-3d-reference-library.html

150. Graphics Language Transmission Format ( $g l T F)$ a File Format Specification for 3D Scenes and Models (Khronos Group, 2021); https://www.khronos.org

151. CCF Registration User Interface (HuBMAP Consortium, 2021); https://hubmapconsortium.github.io/ccf-ui/rui

152. CCF Exploration User Interface (HuBMAP Consortium, 2021); https://portal.hubmapconsortium.org/ccf-eui

153. Human Biomolecular Atlas Program (HuBMAP) Data Portal (NIH, 2020); https://portal.hubmapconsortium.org

154. CCF User Interfaces (RUI, EUI) (HuBMAP Consortium, 2021); https://github.com/hubmapconsortium/ccf-ui

155. Jardine, L. et al. Blood and immune development in human fetal bone marrow and Down syndrome. Nature 598, 327-331 (2021).

156. Stephenson, E. et al. Single-cell multi-omics analysis of the immune response in COVID-19. Nat. Med. 27, 904-916 (2021).

157. Lake, B. B. et al. An atlas of healthy and injured cell states and niches in the human kidney. Preprint at bioRxiv https://doi. org/10.1101/2021.07.28.454201 (2021).

158. Zhuo, L., Huang, L., Yang, Z., Li, G. \& Wang, L. A comprehensive analysis of NPHS1 gene mutations in patients with sporadic focal segmental glomerulosclerosis. BMC Med. Genet. 20, 111 (2019).

159. Ong, E. et al. Modelling kidney disease using ontology: insights from the Kidney Precision Medicine Project. Nat. Rev. Nephrol. 16, 686-696 (2020).

160. Barwinska, D. et al. Molecular characterization of the human kidney interstitium in health and disease. Sci. Adv. 7, eabd3359 (2021).

161. ASCT+B Reporter (HuBMAP Consortium, 2021); https:// hubmapconsortium.github.io/ccf-asct-reporter

162. Majumder, P. P., Mhlanga, M. M. \& Shalek, A. K. The Human Cell Atlas and equity: lessons learned. Nat. Med. 26, 1509-1511 (2020).

163. Spatial Biology Europe: Online (HuBMAP Consortium, 2021); https://cns-iu. github.io/workshops/2021-2004-2014 spatial biology europe online

164. Azimuth App for reference-based single-cell analysis (Satija Lab, 2021); https://azimuth.hubmapconsortium.org/

165. Hao, Y. et al. Integrated analysis of multimodal single-cell data. Cell 184, 3573-3587 (2021)

166. Osumi-Sutherland, D. et al. Cell type ontologies of the Human Cell Atlas. Nat. Cell Biol. https://doi.org/10.1038/s41556-021-00787-7 (2021).

167. Radtke, A. J. et al. IBEX: an open and extensible method for high content multiplex imaging of diverse tissues. Preprint at https://arxiv.org/abs/ 2107.11364 (2021)

168. Hickey, J. W. et al. Spatial mapping of protein composition and tissue organization: a primer for multiplexed antibody-based imaging. Preprint at https://arxiv.org/abs/2107.07953 (2021).

169. Manz, T. et al. Viv: multiscale visualization of high-resolution multiplexed bioimaging data on the web. Preprint at OSF https://doi.org/10.31219/osf.io/ wd2gu (2020).

170. Balhoff, J. \& Curtis, C. K. Ubergraph (2021); https://github.com/ INCATools/ubergraph

171. Li, W., Germain, R. N. \& Gerner, M. Y. Multiplex, quantitative cellular analysis in large tissue volumes with clearing-enhanced 3D microscopy (Ce3D). Proc. Natl Acad. Sci. USA 114, E7321-E7330 (2017).

172. Fedorov, A. et al. 3D Slicer as an image computing platform for the Quantitative Imaging Network. Magn. Reson. Imaging 30, 1323-1341 (2012).

173. Slicer Community. 3D Slicer image computing platform (2021); https://www.slicer.org/

174. ZBrush, the all-in-one digital sculpting solution (Pixologic, 2021); https://pixologic.com/

175. Maya: 3D computer animation, modeling, simulation, and rendering software (Autodesk, 2021); https://www.autodesk.com/products/maya/overview

\section{Acknowledgements}

We thank B. B. Lake from University of California, San Diego, for assistance with annotations and analysing the single-nucleus RNA-seq HUBMAP data for several of the markers in the kidney ASCT+B tables; B. Steck and R. Dull from the University of Michigan for assistance with the nomenclature and curation of kidney partonomy; S. 
Winfree, IUPUI, for discussions regarding the kidney ASCT+B table; and staff at the KPMP, especially the Tissue Interrogation Sites and the Controlled Cell Vocabulary working group, for guidance and development of the initial sets of ASCT+B kidney tables. We acknowledge L. Yao for segmenting and optimizing the mouse popliteal lymph node model from high-resolution microscopy data. The work was funded, in part, by NIH Awards OT2OD026671, U54DK120058, 1UH3CA246594, 1U54AI142766, 1UG3CA256960, 1UG3HL145609, U54HL145608, U54HL145611, UH3DK114933, DK110814, DK107350, U54HG010426, 1R01HL137811, U01DK107350, U24DK110814 U01HL148861, 1U01HL148867, the NIDDK Kidney Precision Medicine Project grant U2CDK114886, the Intramural Research Program of the National Institute of Allergy and Infectious Diseases (NIAID), Department of Health and Human Services under BCBB Support Services Contract HHSN316201300006W/HHSN27200002, Helmsley Charitable Trust 2018PG-T1D071 and 2004-0381, the Allen Institute, Wellcome-Sanger WT206194, and the Chan Zuckerberg Initiative CZF2019-002442.

\section{Competing interests}

In the past 3 years, S.A.T. has received remuneration for consulting and Scientific Advisory Board membership from Genentech, Roche, Biogen, GlaxoSmithKline,
Foresite labs, Qiagen and Transition Bio and she is a co-founder and equity holder of Transition Bio. R.M. receives research funding from Bayer and Amgen and serves as a consultant for Myokardia/BMS and Third Pole; he is a co-founder of Patch Inc; he is a co-inventor for a patent no. PCT/US2O12/O22119 on pharmacologic BMP inhibitors (along with Mass General Brigham) for which he receives royalties from Keros Therapeutics, Inc.; he also receives royalties from UpToDate for scientific content authorship. The other authors declare no competing interests.

\section{Additional information}

Supplementary information The online version contains supplementary material available at https://doi.org/10.1038/s41556-021-00788-6.

Correspondence should be addressed to Katy Börner.

Reprints and permissions information is available at www.nature.com/reprints.

Publisher's note Springer Nature remains neutral with regard to jurisdictional claims in published maps and institutional affiliations.

(C) Springer Nature Limited 2021 